\title{
Eosinophilic esophagitis - recognition, diet and drugs
}

\author{
Marietta lacucci MD PhD, Subrata Ghosh MD FRCPC FRCP FRCPE
}

$E^{\mathrm{a}}$ sinophilic esophagitis (EoE) is an immune-mediated disorder triggered by food or other environmental antigens. EoE presents with food impaction and dysphagia in adults. In children, the symptoms may be more diverse including failure to thrive, vomiting, gagging and gastroesophageal reflux-like symptoms. Overall, the prevalence of EoE in the general population is approximately 50 per 100,000 . EoE is mediated by T helper cell 2 cytokines such as interleukin (IL)-4, IL-5 and IL-13, which promote eosinophil recruitment via eotaxin-3 (1).

The diagnosis of EoE depends on the presence of esophageal dysfunction symptoms, the exclusion of gastroesophageal reflux and the presence of 15 or more eosinophils per high-power microscopy field in esophageal biopsy, with the eosinophilia limited to the esophagus. Proton pump inhibitor-responsive EoE is also a recognized subgroup.

Although EoE may present with normal endoscopic findings, characteristic endoscopic features are increasingly being recognized. These include esophageal rings, strictures, narrow-calibre esophagus, linear furrows, white plaques or exudates, and pallor or decreased vasculature. These findings help to diagnose EoE by guiding biopsy decisions and to assess a patient's response to therapy (2). In a systematic review of endoscopic findings from patients with EoE, Kim et al (3) recently demonstrated a high pooled prevalence of at least one endoscopic finding in patients with EoE, although prevalence rates for individual findings varied and could be low. They concluded that individual endoscopic findings had high levels of specificity but low sensitivity, and the predictive values were inadequate for diagnostic purposes. Using high-resolution endoscopy with filter techniques or using confocal endomicroscopy may increase diagnostic yields, but endoscopist education and familiarity with the abnormalities may be crucial in not missing subtle abnormalities (Figure 1).

The medical treatment of EoE is predominantly via use of topical steroids, either swallowed fluticasone or oral viscous budesonide; the latter may be more effective (4). Second-line medical therapies include systemic steroids and the leukotriene antagonist montelukast. Proton pump inhibitors may be useful because some patients are responsive. Esophageal strictures may be dilated at endoscopy followed by medical therapy (1). Such dilation may be as safe as dilating other benign strictures.
Elimination of putative food antigens is an attractive strategy in the management of EoE. In general, these strategies include an elemental diet, a six-food elimination diet (SFED) and targeted-elimination diet. In the current issue of the Canadian Journal of Gastroenterology, Abraham et al (5) (pages 521-524) reported the effect of a gluten-free diet on EoE in children with coexistent celiac disease and EoE. In only one of seven patients, could histological remission of EoE be demonstrated. The pathogenesis of celiac disease and EoE appear to be distinct; it is unlikely that gluten is an incriminating allergen in the majority of patients with EoE. In children with EoE, an elemental diet is widely used. More studies are required both in children and in adults to identify incriminating food antigens or allergens in EoE to develop acceptable dietary therapy for EoE. The elemental diet, which was first used to treat children with Crohn disease, appears to be extremely effective in EoE, but the therapy is expensive and adherence in adults may be problematic. The SFED eliminates the common food allergens milk, wheat, eggs, soy, seafood and nuts. In adults, a SFED for six weeks resulted in symptomatic improvement in $94 \%$ and histological remission in $64 \%$ of pateints, respectively. Based on re-introduction of eliminated foods, wheat and milk were most commonly incriminated in the recurrence of EoE (6). However, in view of the observation by Abraham et al, it appears that gluten is not the incriminating allergen in wheat in the majority of patients. In adults, the skin prick test was poorly predictive of incriminating food items. In children, the skin prick test and atopy patch test were found to have high negative predictive value and, using these tests, led to elimination of fewer food items but comparable efficacy to empirical elimination diets (7).

A combination of topical steroids and dietary therapy in adults has not been systematically tested but may be an attractive option. In view of the fact that viscous topical steroid therapy may be more effective than nebulized steroid therapy in EoE, a change in practice is required - we need to advocate for the availability of such pharmaceutical products. Most physicians in Canada use swallowed aerosolized fluticasone with occasional use of local pharmacy-prepared viscous budesonide - dietary therapy is rarely used in adults. This is an area in which dieticians may have an important role. We need
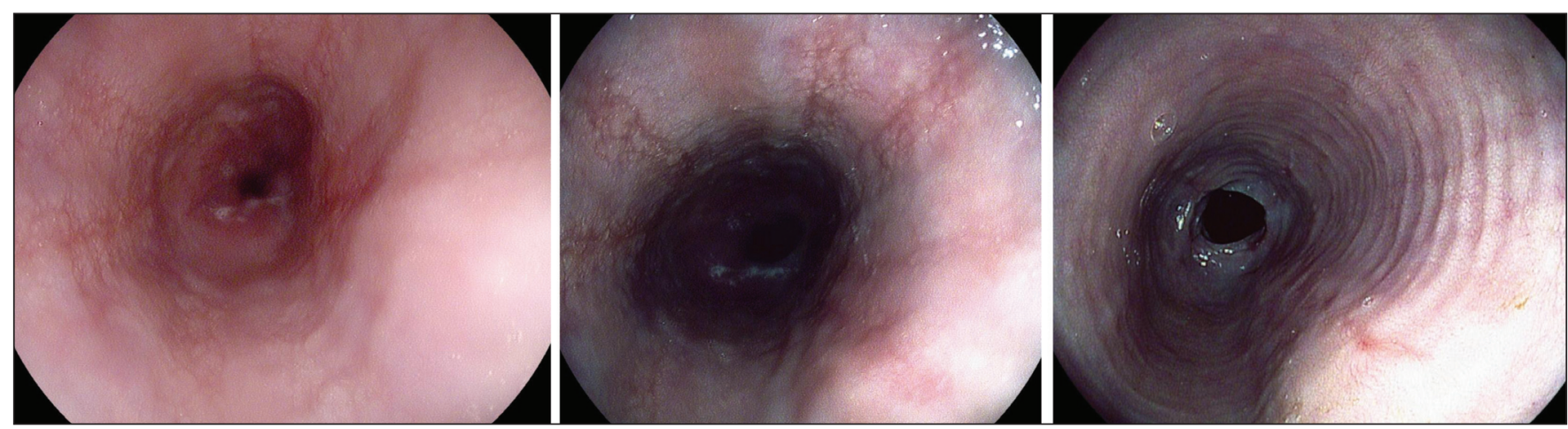

Figure 1) Characteristic endoscopic appearance of eosinophilic esophagitis. Left: Pallor, decreased vasculature and subtle furrows - standard white light endoscopy. Middle: Multiple longitudinal furrows in the esophagus demonstrated with high-definition endoscopy and iScan (Pentax, Japan).

Right: Multiple esophageal rings giving a tracheal ring-like (feline) appearance with high-definition endoscopy and iScan

Division of Gastroenterology, Department of Medicine, University of Calgary, Calgary, Alberta

Correspondence: Dr Subrata Ghosh, Division of Gastroenterology, Department of Medicine, 3280 Hospital Drive Northwest, Calgary,

Alberta T2N 4N1. Telephone 403-944-8222, fax 403-944-1095, e-mail subrata.ghosh@albertahealthservices.ca

Received and accepted for publication July 3, 2012 
Canadian guidance and recommendations regarding uniform diagnostic and therapeutic algorithms both in children and in adults with EoE because every gastroenterologist now should be competent in managing this condition.

\section{REFERENCES}

1. Dellon ES. Diagnosis and management of eosinophilic esophagitis. Clin Gastroenterol Hepatol 2012; doi: 10.1016/j.cgh.2012.06.003.

2. Joo MK, Park JJ, Kim SH, et al. Prevalence and endoscopic features of eosinophilic esophagitis in patients with esophageal or upper gastrointestinal symptoms. J Dig Dis 2012;13:296-303.

3. Kim HP, Vance RB, Shaheen NJ, Dellon ES. The prevalence and diagnostic utility of endoscopic features of eosinophilic esophagitis: A meta-analysis. Clin Gastroenterol Hepatol 2012 May 18 (In press).
4. Dellon ES, Sheikh A, Speck O, et al. Viscous topical is more effective than nebulized steroid therapy for patients with eosinophilic esophagitis. Gastroenterology (in press)

5. Abraham JR, Persad R, Turner JM, Huynh HQ. Gluten-free diet does not appear to induce endoscopic remission of eosinophilic esophagitis in children with coexistant celiac disease.

Can J Gastroenterol 2012;26:521-4.

6. Gonsalves N, Yang G-Y, Doerfler B, Ritz S, Ditto AM, Hirano I. Elimination diet effectively treats eosinophilic esophagitis in adults; food reintroduction identifies causative factors. Gastroenterology 2012;142:1451-9.

7. Spergel JM, Brown-Whitehorn TF, Cianferoni A, et al. Identification of causative foods in children with eosinophilic esophagitis treated with an elimination diet. J Allergy Clin Immunol 2012 (In press). 


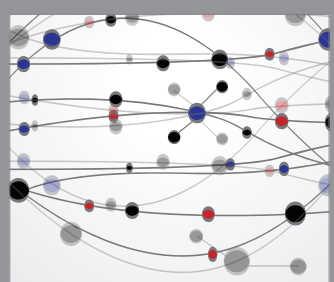

The Scientific World Journal
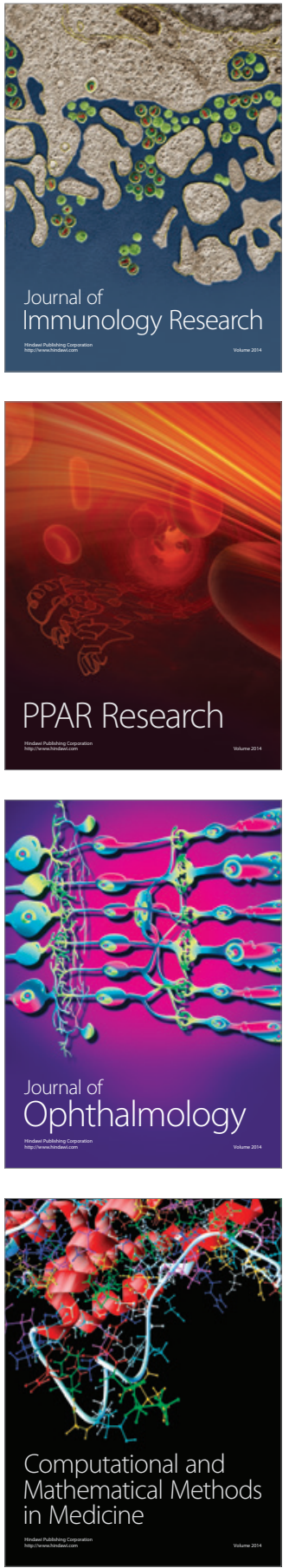

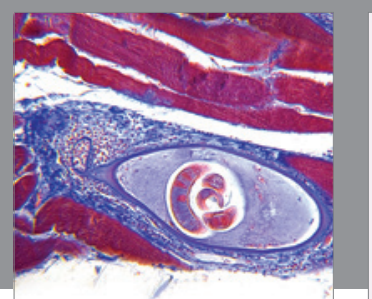

Gastroenterology Research and Practice

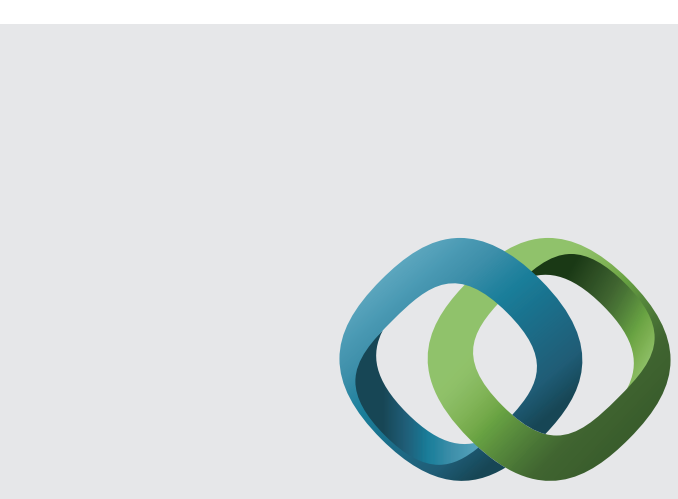

\section{Hindawi}

Submit your manuscripts at

http://www.hindawi.com
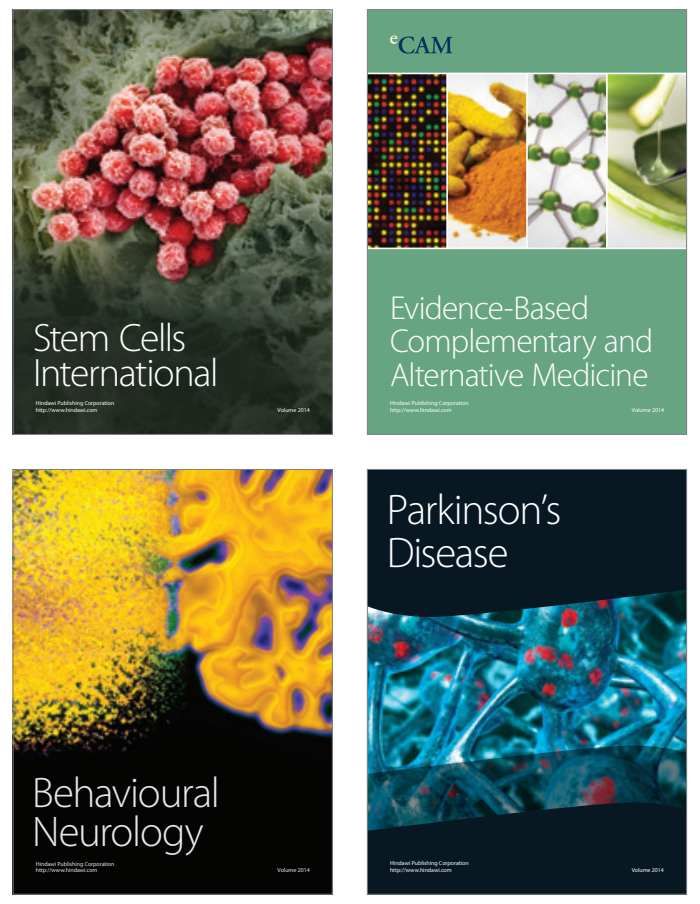
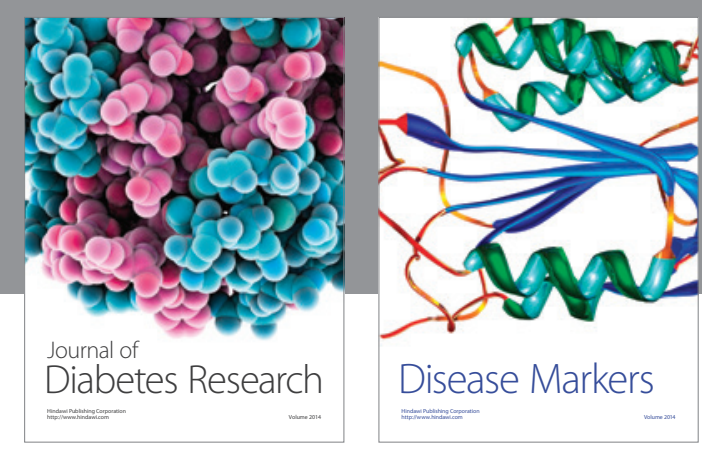

Disease Markers
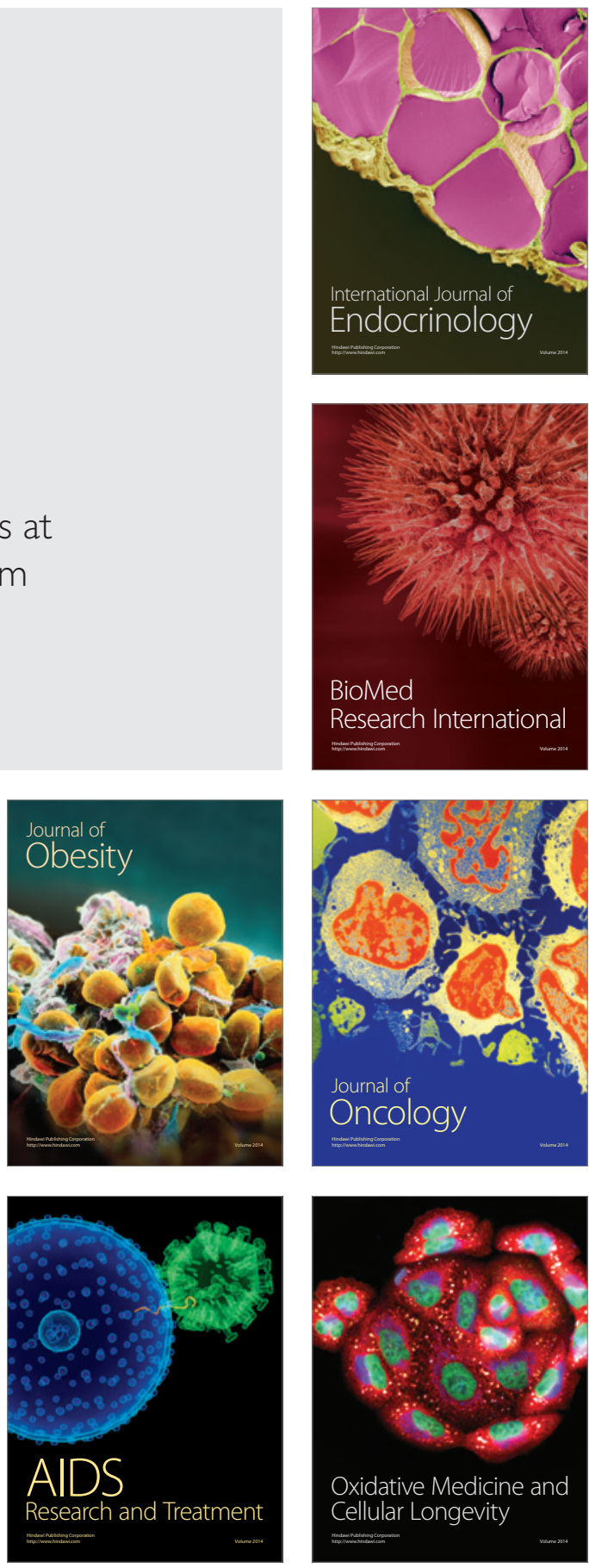